\title{
Ultrasound-Guided Access in a Malrotated Kidney: Optimally Safe and Effective
}

\author{
Aisosa Omorogbe, BS, Daniel C. Rosen, MD, Ryan Chandhoke, MD, \\ Jacob Bamberger, BS, and Mantu Gupta, MD
}

\begin{abstract}
Background: Management of renal calculi in a patient with kidney malrotation can be difficult because of complexity and alteration of collecting system anatomy. Pyelolithotomy, whether open, laparoscopic, or robotic, has been shown to be an effective method of stone removal in this patient population; however, it is not always ideal because of its invasiveness and increased morbidity. Ideally, a percutaneous approach may be less invasive, and if feasible, it can optimize patient safety and stone-free status.

Case Presentation: Here we present a case of a 68-year-old Caucasian female who presented with $2.7 \mathrm{~cm}$ stone in the renal pelvis of a severely malrotated left kidney, which was managed using a combination of fluoroscopy and ultrasound (US)-guided percutaneous nephrolithotomy.

Conclusion: US-guided access properly delineates the anatomic complexities of a severely malrotated kidney and permits safe percutaneous management of large stones. This is because fluoroscopic guidance alone may lead to inadvertent adjacent visceral organ trauma and increased risk of parenchymal and intrarenal vascular injury.
\end{abstract}

Keywords: PCNL, malrotation, ultrasound, calculus, pyelolithotomy, UTI

\section{Clinical History}

A 68-YEAR-OLD FEMALE with a medical history significant for recurrent urinary tract infections (UTIs) on chronic antibiotics, hypothyroidism, hypertension, hyperlipidemia, prediabetes, and a 50-pack-year smoking history had an MRI at an outside hospital for a possible liver hemangioma, which incidentally found a severely malrotated left kidney with a $2.7 \mathrm{~cm}$ calculus in the renal pelvis, marked hydronephrosis and cortical scarring (Fig. 1).

A CT was done that confirmed the MRI diagnosis (Fig. 2), and she was scheduled for a laparoscopic left pyelolithotomy. She was concerned about the potential morbidity of the procedure and presented for a second opinion to our institution with a complaint of sporadic left flank pain and persistently cloudy urine. Urine culture was positive for Escherichia coli, for which she was treated. She then underwent a retrograde pyelogram (RPG) in the office to better evaluate ureteral and renal anatomy.

\section{Diagnosis}

Left RPG demonstrated a tortuous ureter that required manipulation to access the proximal ureter, up to the malrotated renal pelvis. Fluoroscopy revealed a large round
$2.7 \mathrm{~cm}$ calculus in the renal pelvis of a severely malrotated left kidney with a thick halo of filling defect around the stone, indicating either edema/impaction or nonradiopaque purulent material surrounding the stone (Fig. 3). The renal pelvis was more anterior, inferior, and lateral than an anatomically placed kidney. Options for stone management were discussed with the patient. Given the size of the stone, she was consented for percutaneous removal of left renal calculus.

\section{Intervention}

The patient was started on culture-specific antibiotics (trimethroprim-sulfamethoxazole) 10 days before the procedure. She was placed in the prone split leg position on spreader bars. Prone flexible cystoscopy was performed with guidewire placement and placement of a $5 \mathrm{~F}$ ureteral catheter into the renal pelvis. Contrast was injected for retrograde ureteropyelography that showed the known large calculus and tortuous ureter because of the kidney malrotation along with severe hydronephrosis (Fig. 4). Ultrasound (US) was used to observe the stone as well as any other surrounding organs to ensure a safe access tract in this patient with abnormal anatomy. The stone was observed, but direct access in the usual location from posterolateral would not go through any parenchyma; therefore, the US probe was reversed to create a more

Department of Urology, Icahn School of Medicine at Mount Sinai, New York, New York, USA. 

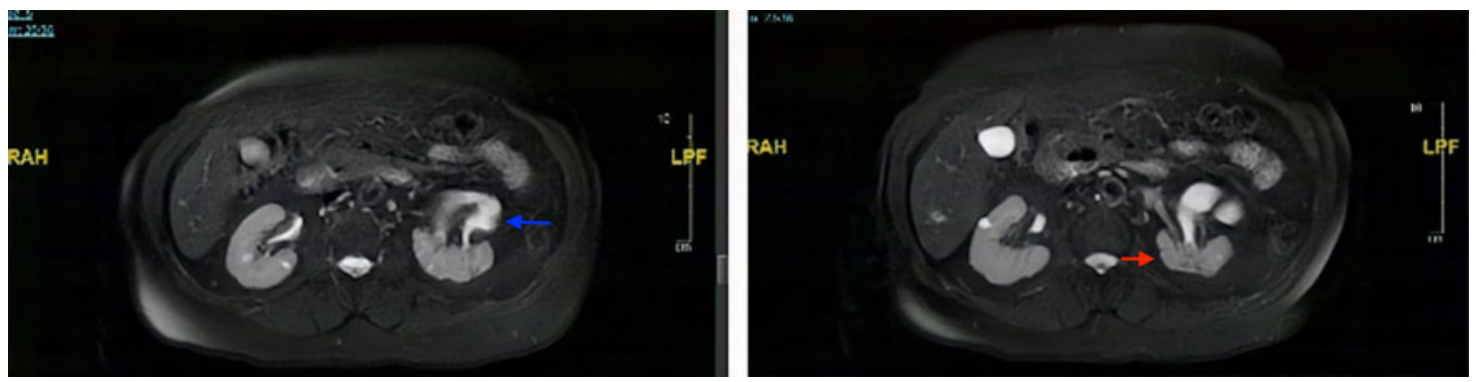

FIG. 1. Selected axial MRI showing left malrotated kidney (red arrow) with severe hydronephrosis (blue arrow).
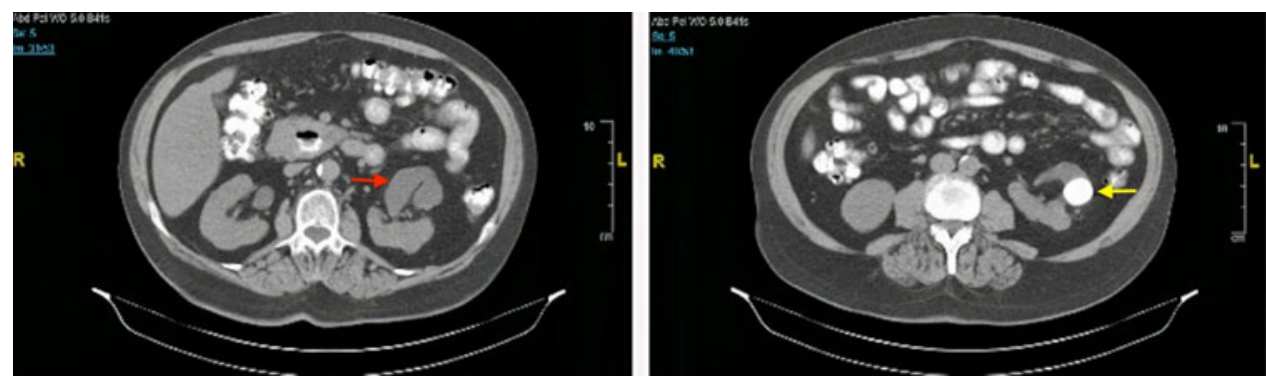

FIG. 2. Axial CT of malrotated left kidney with hydronephrosis of tortuous ureter (red arrow) and $2.7 \mathrm{~cm}$ stone (yellow arrow).
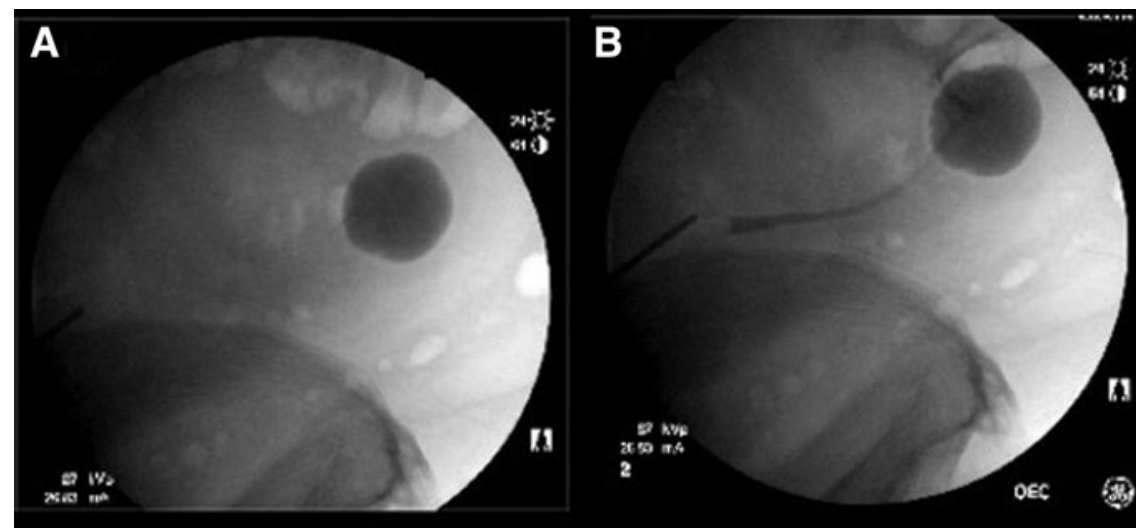

C

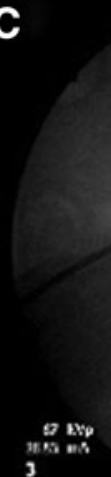

D

E
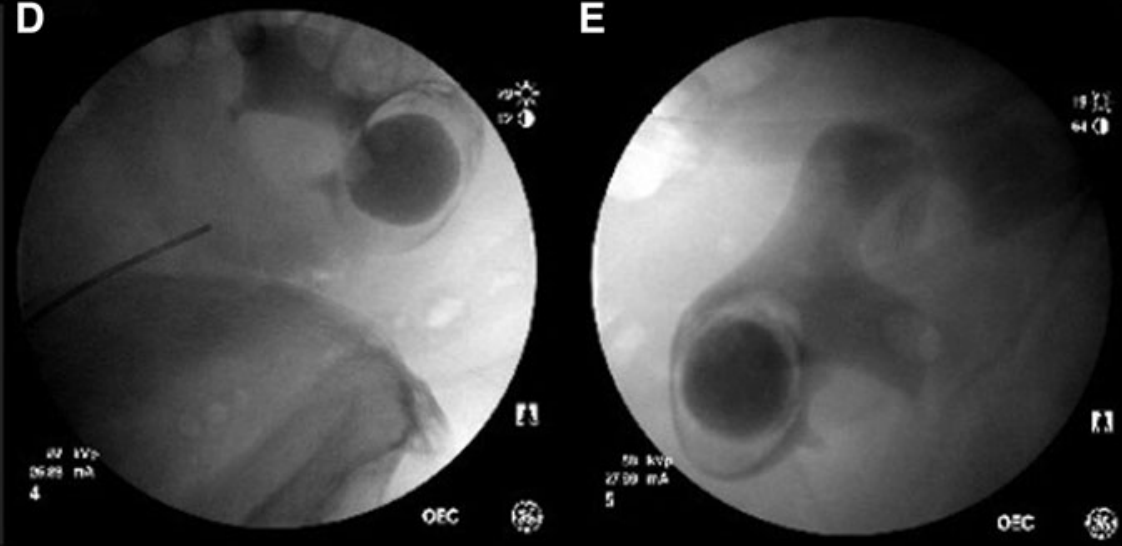

FIG. 3. Fluoroscopy showing stone in the renal pelvis of a malrotated kidney. (A) Scout film of stone (B, C) retrograde pyelogram showing catheter in proximal ureter and malrotated collecting system with stone in renal pelvis. (D) Contrast surrounding stone with large halo of filling defect indicating edema/impaction or purulent material. (E) Prone film of retrograde pyelogram. 


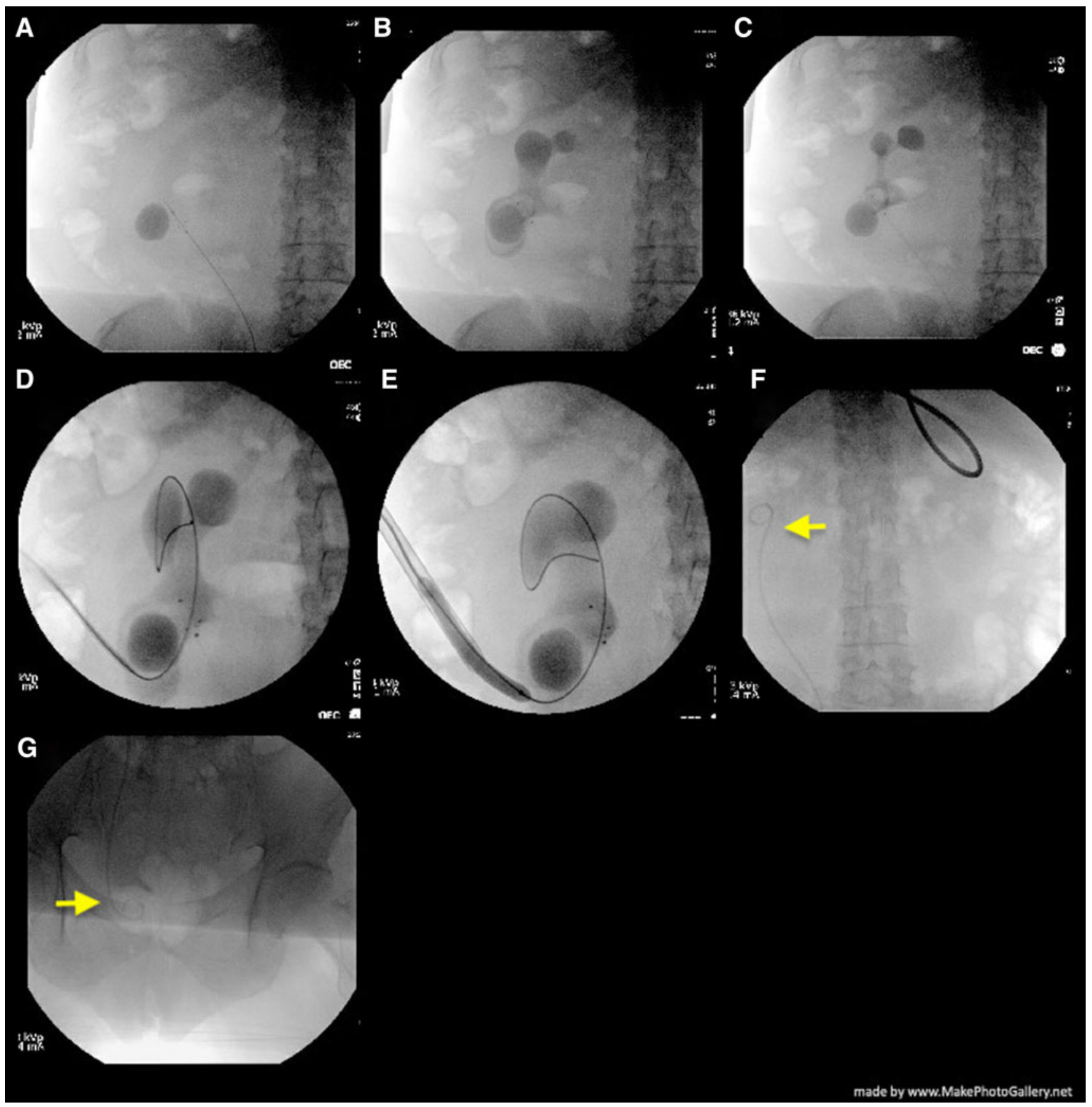

FIG. 4. Fluoroscopy depicting superior access to the malrotated kidney using a "top-down" approach through parenchyma to reduce chance of collecting system injury (A-E). Ureteral stent placement from the renal pelvis $(\mathbf{F})$ into the bladder (G).

anterior top-down trajectory through the parenchyma and safe from surrounding organs. Access was obtained solely with US guidance using a $20 \mathrm{~cm}$ EchoTip ${ }^{\circledR}$ needle (Cook Urological, Spencer, IN). Under fluoroscopic guidance, the tract was dilated with a $24 \mathrm{~F}$ NephroMax ${ }^{\circledR} \mathrm{TM}$ (Boston Scientific, Marlborough, MA) balloon device into the collecting system. The stone was identified with a thick surrounding rim of bacterial biofilm. The substance was suctioned and the stone was fragmented with both ultrasonic and ballistic energy.

Once stone free, a ureteral stent was placed antegrade from the renal pelvis into the bladder along with a separate ne- phrostomy catheter for drainage (Fig. 4). The patient was kept on broad spectrum antibiotics and laboratory values were stable on postoperative day 1 , and she was subsequently discharged with a Foley catheter in place and cultureappropriate antibiotics.

\section{Follow-Up}

The patient returned to clinic on postoperative day 5 for nephrostomy removal. Stone analysis was mixed with $50 \%$ calcium oxalate monohydrate, $25 \%$ calcium oxalate dihydrate, 
and $25 \%$ carbonate apatite. Stone culture was positive for E. coli. She was started on a 6-month course of trimethroprimsulfamethoxazole for secondary prophylaxis. She returned to our office 10 days later for left ureteral stent removal. Followup ultrasonography at 2 months postoperatively shows the patient to be stone free without urinoma or abscess. She has done well without flank pain or UTIs since surgery.

\section{Discussion}

Kidney malrotation is a developmental anomaly that occurs as the kidneys rotate and ascend from the true pelvis to the renal fossa during the 6th-9th week of development. This results in an anterolateral facing renal pelvis, posteromedial facing renal calices, vascular and collecting system anomalies, and altered relation to abdominal organs. ${ }^{1}$ The altered orientation of the kidney can make stone removal by percutaneous nephrolithotomy (PCNL) difficult, but studies have also shown that it can be a safe procedure as long as careful preoperative and postoperative planning is undertaken. ${ }^{2-4}$ Alternatively, pyelolithotomy may be considered, which can take advantage of the anterior facing renal pelvis, but is more invasive and morbid, with concomitant risk for abdominal injury. Other viable options for stone removal in malrotated kidneys include flexible ureteroscopic lithotripsy and mini-PCNL; however, because of the significantly large stone burden, severe hydronephrosis, and anatomic complexity of our patient, these methods were not pursued further.

This case report adds to the growing literature on the safety and efficacy of minimally invasive techniques in the management of an anomalous kidney with significant stone disease. When determining ideal access that prevents injury to surrounding organs and the collecting system, a complex anatomy must be taken into account to render the patient stone free. US-guided percutaneous access provides realtime visualization of the malrotated anatomy and the intervening structures between the skin and the kidney, enabling optimal access, which would not be feasible with fluoroscopic guidance alone. After stent removal, our patient has had no complications; however, based on her chronically dilated collecting system and metabolic risk factors, compliance to postoperative antibiotics and antilithogenic regimen will be necessary to prevent infection and disease recurrence.

\section{Disclosure Statement}

No competing financial interests exist.

\section{Funding Information}

No funding was received for this article.

\section{References}

1. Lim TJ, Choi SK, You HW, Kim MJ, Ahn JS, Kim TG, Kim JI, Jeon SH. Renal cell carcinoma in a right malrotated kidney. Korean J Urol 2011;52:792-794.

2. Prakash G, Sinha RJ, Jhanwar A, Bansal A, Singh V. Outcome of percutaneous nephrolithotomy in anomalous kidney: Is it different? Urol Ann 2017;9:23-26.

3. Sabler IM, Katafigiotis I, Gofrit ON, Duvdevani M. Present indications and techniques of percutaneous nephrolithotomy: What the future holds? Asian J Urol 2018;5:287-294.

4. Mosavi-Bahar SH, Amirzargar MA, Rahnavardi M, Moghaddam SM, Babbolhavaeji H, Amirhasani S. Percutaneous nephrolithotomy in patients with kidney malformations. J Endourol 2007;21:520-524.

$$
\begin{array}{r}
\text { Address correspondence to: } \\
\text { Aisosa Omorogbe, BS } \\
\text { Department of Urology } \\
\text { Icahn School of Medicine at Mount Sinai } \\
1 \text { Gustave L. Levy Pl } \\
\text { New York, NY } 10029
\end{array}
$$

USA

E-mail: aisosa.omorogbe@icahn.mssm.edu

$\begin{aligned} & \text { Abbreviations Used } \\ \mathrm{CT} & =\text { computed tomography } \\ \mathrm{MRI} & =\text { magnetic resonance imaging } \\ \mathrm{PCNL} & =\text { percutaneous nephrolithotomy } \\ \mathrm{RPG} & =\text { retrograde pyelogram } \\ \mathrm{US} & =\text { ultrasound } \\ \mathrm{UTI} & =\text { urinary tract infection }\end{aligned}$

Cite this article as: Omorogbe A, Rosen DC, Chandhoke R, Bamberger J, Gupta M (2020) Ultrasound-guided access in a malrotated kidney: Optimally safe and effective, Journal of Endourology Case Reports 6:3, 166-169, DOI: 10.1089/cren.2019.0155. 